\title{
Comparison of the Performance Evaluation of the MicroPET R4 Scanner According to NEMA Standards NU 4-2008 and NU 2-2001
}

\author{
Fotini D. Popota, Pablo Aguiar, J. Raúl Herance, Deborah Pareto, Santiago \\ Rojas, Domènec Ros, Javier Pavía and Juan Domingo Gispert
}

\begin{abstract}
The purpose of this work was to evaluate the performance of the microPET R4 system for rodents according to the NU 4-2008 standards of the National Electrical Manufacturers Association (NEMA) for small animal PET systems and also to compare its performance evaluation against its previous evaluation according the adapted clinical NEMA NU 2-2001. The performance parameters evaluated here were spatial resolution, sensitivity, scatter fraction, counting rates for rat- and mouse-sized phantoms and image quality. Spatial resolution and sensitivity were measured with a ${ }^{22} \mathrm{Na}$ point source, while scatter fraction and count rate performance were determined using a mouse and rat phantoms with an ${ }^{18} \mathrm{~F}$ line source. The image quality of the system was assessed using the NEMA image quality phantom. Assessment of attenuation correction was performed using $\gamma$-ray transmission and CTbased attenuation correction methods. At the center of the field of view, a spatial resolution of $\mathbf{2 . 1 2} \mathbf{~ m m}$ at full width half maximum (FWHM) (radial), $2.66 \mathrm{~mm}$ FWHM (tangential) and $2.23 \mathrm{~mm}$ FWHM (axial) was measured. The absolute sensitivity was found to be $1.9 \%$ at the center of the scanner. Scatter fraction for mouse-sized phantoms was $8.5 \%$ and the peak count rate was $311 \mathrm{kcps}$ at $153.5 \mathrm{MBq}$. The rat scatter fraction was $22 \%$ and the peak count rate was $117 \mathrm{kcps}$ at $123.24 \mathrm{MBq}$. Image uniformity showed better results with 2D FBP, while an overestimation of the recovery coefficients was observed when using 2D and 3D OSEM MAP reconstruction algorithm. All measurements were made for an energy window of 350-650 keV and a coincidence window of 6 ns. Histogramming and reconstruction parameters were used according to the manufacturer's recommendations. The microPET R4 scanner was fully characterized according to the NEMA NU 4-2008 standards. Our results diverge considerably from those previously reported with an adapted version of the NEMA NU 2-2001 clinical standards. These discrepancies can be attributed to the modifications in NEMA methodology, thereby highlighting the relevance of specific small animal standards for the performance evaluation of PET systems.
\end{abstract}

Index Terms - NEMA, spatial resolution, scatter fraction, uniformity

Manuscript received April 12, 2012; revised June 28, 2012; accepted July 10, 2012. Date of publication August 20, 2012; date of current version October 09, 2012. This work was supported in part by the Fondo de Investigación Sanitaria (FIS) of the Instituto de Salud Carlos III under Grants PS09/02620 and PS09/ 02217, the CDTI as part of the CENIT Program (AMIT Project), the Ministerio de Ciencia e Innovación under Project No. SAF2009-08076, and the Spanish Ministry of Economy and Competitiveness. 
F. D. Popota is with the Institut d'Alta Tecnologia-PRBB, CRC Corporacio Sanitaria Barcelona, CO 08003, Spain, and also with the Unitat de Biofisica I Bioenginyeria, Universitat de Barcelona, Barcelona 08036, Spain. P. Aguiar is with theMolecular Imaging Group, Foundacion IDICHUS/IDIS,

Santiago de Compostela 15706, Spain. D. Pareto, R. Herance, S. Rojas, and J. D. Gispert are with the Institut d'Alta Tecnologia-PRBB, CRC Corporacio Sanitaria Barcelona, Barcelona

08003, Spain, and also with the CIBER en Bioengenier'1a, Biomateriales y Nanomedicina (CIBER BBN), Barcelona 50018, Spain (e-mail: jdgispert@fpmaragall. org). D. Ros is with the Unitat de Biofisica i Bioenginyeria, Universitat de Barcelona, Barcelona 08036, Spain, and also with the CIBER en Bioingenieria, Biomateriales y Nanomedicina, Zaragoza 50018, Spain, and the Institut d'Investigacions Biomediques Agust Pi i Sunyer (IDIBAPS), Barcelona 08036, Spain. J. Pavia is with the CIBER en Bioingenieria, Biomateriales y Nanomedicina, Zaragoza, Spain, and also with the Servei deMedicina Nuclear, Hospital Clínic, IDIBAPS, Barcelona 08036, Spain Digital Object Identifier 10.1109/TNS.2012.2208760

\section{INTRODUCTION}

OVER the last two decades positron emission tomography (PET) has been used to measure concentrations of positron emitter ligands for diagnostic purposes, to evaluate therapeutic agents and to provide insight into disease biology. Applied to laboratory animals, this technique permits the use of experimental approaches impracticable for humans and constitutes a powerful tool for translational research. Small animal PET applications range from investigations of receptor-ligand interactions, metabolism, gene expression, cell therapy, development of new drugs [1] and cell proliferation and migration [2]. With the use of these dedicated systems, molecular biology and in vivo imaging were intersected so as to establish molecular imaging [3]. The visualisation and follow-up of all molecular processes in living organisms has opened up huge potential in the fields of oncology, neurological and cardiovascular diseases [4].

As new probes are being developed, technical aspects are becoming more relevant depending on the specific application. Due to the significant difference in size compared to humans, small animal PET systems face the challenge of achieving suitable sensitivity and spatial resolution without any increased cost. PET detector technology has thus pushed the limits to detect and accurately quantify nanomolar or picomolar concentrations of molecular probes. Since the first generation of animal scanners [5], [6] many other systems have been developed and made commercially available [7] - [11]. This made preclinical PET systems popular for a large number of animal imaging studies because they reduced the cost of detectors and electronics.

The last few years, many efforts have been made to improve system performance by enhancing organ uptake quantification and providing images with quality equivalent to those of humans. The knowledge of the physical characteristics of each system, expressed in terms of spatial resolution, sensitivity, scatter fraction and count rate performance, image quality and accuracy of corrections, is the key point when making decisions 
about the most appropriate scanner to be used for particular experiments.

Scanners differ in design and characteristics and as a consequence, a standardization of acquisition protocols and reconstruction methods is needed to compare their performances. Thus to optimize the capabilities of small animal PET scanners, their performance has to be evaluated according to a standardized methodology. Until recently, the clinical standards of the National Electrical Manufacturers Association (NEMA), such as NEMA NU 2-1994 [12] or NEMA NU 2-2001 [13], were adapted for small animal PET scanners. For example, the microPET P4 [10] and the quad-HIDAC [14] were characterized according to human clinical NEMA standards but their performance using non-standardized phantoms and experimental procedures may lead to variations on the results provided.

The NEMA published its NEMA NU 4-2008 standards, which provide a full protocol for the performance evaluation of small animal PET scanners [15]. So far, not many scanners have been evaluated according to these standards apart from the Inveon preclinical tomograph [16], the FLEX Triumph X-PET scanner [17], the Mini PET II small animal scanner [18], the microPET Focus 120 scanner [19] and the coplanar multimodality scanner for rodent imaging [20].

The microPET R4 system (Concorde Microsystems Inc.) is a dedicated system for imaging mice and rats. It has been one of the first small animal PET scanners with an axial field of view (FOV), which provides wholebody scans of rodents that permit longitudinal studies. Its performance has previously been evaluated by Knoess C, et al [21] according to the NEMA NU 2-2001 for clinical PET scanners.

In this work, we report the performance evaluation of the microPET R4 scanner using the recently published NEMA NU-4 standards for small animal PET systems. The scope of this standard is to provide a standardized methodology for the evaluation of the performance of small animal PET systems which did not exist up to now and evaluations were done adopting clinical PET standards. Therefore, one of our primary goals was to assess any significant differences between the results obtained with NEMA NU 22001 and NEMA NU 4-2008 that which would justify any possible reevaluation of small animal PET systems.

\section{MATERIALS AND METHODS}

\section{A. System Description}

The microPET R4 scanner is a dedicated PET system for imaging rodents. The scanner's detectors are made of $\mathrm{Lu} 2(1-\mathrm{x}) \mathrm{Ce} 2 \mathrm{x}(\mathrm{SiO} 4) \mathrm{O}-$ (LSO), which has high stopping power for $511 \mathrm{keV}$ photons, a high light output and a fast decay time of $40 \mathrm{~ns}$ [22], [23]. A scintillation block of $19 \times 19 \times 10 \mathrm{~mm}^{3}$ is sawed to an $8 \times 8$ crystal array with $9 \mathrm{~mm}$ depth cuts, 
leaving a $1 \mathrm{~mm}$ thick layer of LSO at the bottom to hold the block. The crystal size is $2.1 \times 2.1 \times 10 \mathrm{~mm}^{3}$ with an axial and transaxial pitch of 2.423 $\mathrm{mm}$ and $2.423 \mathrm{~mm}$, respectively, and the cuts are filled with a reflective material after polishing [24]. Each block is coupled to a position sensitive photomultiplier (PS-PMT, Hamamatsu R5900-C8) via a $10 \mathrm{~cm}$ fiber optic bundle. Four blocks of detector comprise one axial detector module, providing 32 detector rings in total. The scanner's diameter is $148 \mathrm{~mm}$, the axial length is $78 \mathrm{~mm}$ and the effective FOV is restricted to $100 \mathrm{~mm}$ transaxially. A coincidence timing window of $6 \mathrm{~ns}$ and a 350-650 keV energy window were used for all the measurements. The average energy resolution of the system is $23 \%$ [21].

The performance characteristics of the system that were evaluated here, following the NEMA NU-4 2008 standards, were the spatial resolution, sensitivity, scatter fraction, counting rates and image quality. A brief description of the experiments is provided below for each parameter.

\section{B. Spatial Resolution}

The spatial resolution of the system is the ability to distinguish between two points after image reconstruction. This is typically measured by the width of the reconstructed point spread function of a point source, and the degree of spreading (blurring) of the point object is a measure for the quality of the imaging system. For these measurements a $1-\mathrm{mm}^{22} \mathrm{Na}$ point source of $166 \mathrm{kBq}$ was placed at various radial distances $(5,10,15$ and $25 \mathrm{~mm}$ ) from the axial center of the FOV and at $1 / 4$ of the axial FOV. Data were acquired for $60 \mathrm{~s}$ in order to collect at least 10,0000 prompt counts. Listmode data of each location were histogrammed into 3D sinograms which were rebinned into 2D sinograms using Fourier rebinning with a span of 3 and a ring difference of 31, which is the maximum ring difference (MRD) of the microPET R4 scanner. The spatial resolution was determined by forming one-dimensional response functions through the peak of the image volume in three orthogonal directions. The image pixel size was made one fifth of the expected full width half maximum (FWHM) in the transverse dimensions.

The volumetric resolution of the system was also calculated here by the product of the three components of the spatial resolution; i.e. radial, tangential and axial resolution. Although NEMA standards do not include this calculation, we consider it a means to indicate the element with the smallest volume that can be resolved accurately with the device.

\section{Scatter fraction, count losses and random coincidence measurements}

The purpose here was to measure the relative system sensitivity to scattered radiation and the effects of dead time and random events at 
various levels of activity. We used two cylindrical high density polyethylene phantoms representing rodents; mice and rats. The mousesized phantom was $25 \mathrm{~mm} \pm 0.5 \mathrm{~mm}$ diameter and $70 \mathrm{~mm} \pm 0.5 \mathrm{~mm}$ long. A cylindrical hole of $3.2 \mathrm{~mm}$ was drilled parallel to the center axis, at a radial distance of $10 \mathrm{~mm}$. The rat-sized phantom was $50 \mathrm{~mm} \pm 0.5 \mathrm{~mm}$ diameter and $150 \mathrm{~mm} \pm 0.5 \mathrm{~mm}$ long, with a cylindrical hole drilled at a radial distance of $17.5 \mathrm{~mm}$. Line sources were $10 \mathrm{~mm}$ shorter than the cylindrical phantoms. Both phantoms were centered in the axial and transaxial direction of the scanner. The initial activity for the mouse-sized and rat-sized phantom was $8.5 \mathrm{mCi}(314.5 \mathrm{MBq})$ and $6.5 \mathrm{mCi}(240.5 \mathrm{MBq})$ [18F]-FDG respectively. Since the microPET R4 system presents intrinsic radioactivity $\left({ }^{176} \mathrm{Lu}\right)$, a measurement of the intrinsic true count rate was also made without any activity in the test phantom line source. The duration of each frame was $1.600 \mathrm{~s}$ with a total acquisition time of $43.200 \mathrm{~s}$. The total event rate, true event rate, random coincidences, scatter coincidences and noise equivalent count (NEC) rate were calculated as a function of the phantom activity. Furthermore, the scatter fraction of the system was calculated by the last frames due to the low activity concentration in the FOV, with count-loss rates and random-event rates below $1.0 \%$ of the true event rate. No corrections of scatter, random, dead time or attenuation were performed. Single slice rebinning (SSRB) was performed in 3D sinograms to obtain 2D data sets.

\section{Sensitivity}

The ability of the scanner to detect positron annihilation $\gamma$-rays was measured as a fraction of the coincidence photon pairs emitted from the source and detected by the equipment. This is an important parameter that needs to be evaluated for each system especially when using dynamic imaging with frames of short duration or tracers that can only be injected in extremely low amounts, such as [11C]- Carfentanil [25].

We used the same ${ }^{22} \mathrm{Na}$ point source as in the case of spatial resolution. The source was placed in the center of the FOV axially and trasaxially. Acquisitions were performed for $60 \mathrm{~s}$ at each position while the source was stepped axially to the either end of the scanner's FOV so as to collect 10,000 true events. The step size was identical to the thickness of the sinogram slice; $1.21 \mathrm{~mm}$. Background true event rates were also determined by acquiring a dataset with no source in the FOV for $60 \mathrm{~s}$. SSRB was used to assign counts in oblique LORs to the image slice where the LOR crosses the scanner axis, so that each slice is represented by one sinogram. For each row of the sinogram, the highest value was located and all pixels greater than $1 \mathrm{~cm}$ from this peak value were set to zero. Sensitivity for each slice was calculated by dividing the total counts of each slice by the total activity of the source, correcting for the branching ratio of ${ }^{22} \mathrm{Na}$. 


\section{E. Image Quality}

The purpose of this measurement was to produce images simulating those obtained in a total body imaging study of a small rodent with hot lesions and with uniform hot and cold areas. Three different measurements are computed to describe different quality aspects of reconstructed images: i) uniformity, as a measure of statistical noise in both hot and cold regions; ii) the recovery coefficient, which reflects quantitative accuracy and is known to be highly influenced by spatial resolution in small objects; and iii) spill - over ratio as another indication of the spatial resolution achieved for the images.

A NEMA phantom was specifically designed to perform these measurements. This consists of three main parts made of polymethylmethacrylate with internal dimensions of $50 \mathrm{~mm}$ in length and $30 \mathrm{~mm}$ in diameter. The main phantom body was composed of a fillable cylindrical chamber with $30 \mathrm{~mm}$ diameter and $30 \mathrm{~mm}$ length. The remaining $20 \mathrm{~mm}$ of the length of the phantom body was solid, with 5 fillable rods with a diameter of 1, 2, 3, 4 and $5 \mathrm{~mm}$ each. A lid attached to the large uniform end region of the phantom supported the two cold region chambers. One of these chambers was filled with non-radioactive water and the other was left with air inside. Both these chambers were composed of hollow cylinders of $15 \mathrm{~mm}$ in length and $8 \mathrm{~mm}$ in internal diameter, $10 \mathrm{~mm}$ in outer diameter and $1 \mathrm{~mm}$ wall thickness. The whole phantom was filled with $114 \mu \mathrm{Ci} 18 \mathrm{~F}-\mathrm{FDG}$ for a 20 min acquisition scan. The phantom was placed on the tomograph bed in such a way that the axis of its main cylindrical compartment was aligned with the axis of the tomography FOV.

We evaluated two methods to correct attenuation of the data; a radionuclide-based transmission scan and an X-ray CT-based transmission scan. NEMA standards do not specify a radionuclide-based transmission scan but in the case of the microPET R4 this is the method provided by the manufacturer. A 68Ge line source was used through a transmission scan so as to measure the attenuation along all the lines of responses (LOR). We also performed a reference scan, called blank scan, before the transmission scan to ensure that the ratio of the blank counts to the transmission counts yielded a correction factor for each emission LOR. Images were reconstructed to pixel sizes of $0.84 \mathrm{~mm}$ (radially and transaxially) using 2D FBP with no axial filter, FORE+2D+OSEM with 4 iterations and 16 subsets, and finally 3D-OSEM with 12 subsets, 2 iterations and 18 MAP iterations. All reconstruction parameters were left to their default values, which are the ones recommended by the scanner's manufacturer.

CT scan was obtained by using the Discovery ST PET/CT (GE) system with a tube voltage of $120 \mathrm{kV}$ and $100 \mathrm{~mA}$. Images were reconstructed with a voxel size of $0.1875 \times 0.1875 \times 0.625 \mathrm{~mm} 3$ providing an image volume of 
$512 \times 512 \times 153 \mathrm{~mm} 3$. The CT image was down-sampled to the microPET image bin size using the software provided with the microPET R4 scanner. The same software was used to convert CT Hounsfield numbers to linear attenuation coefficients at $511 \mathrm{keV}$. Different regions representing different tissues were segmented and then assigned with the appropriate $\mu$-value. An attenuation sinogram was subsequently created using the attenuation wizard of the microPET R 4 and a re-scale of the $\mu$-map image to the actual $\mu$-values and segmentation by thresholding of the $\mu$-values took place. The next step was to perform Fourier rebinning for the same span and ring difference as the microPET images.

The data evaluated here after the reconstruction of the image, were the image uniformity, recovery coefficient values and accuracy of corrections. Uniformity was calculated by applying a volume of interest (VOI) of 22.5 $\mathrm{mm}$ in diameter by $10 \mathrm{~mm}$ in length over the center of the uniform region of the image quality phantom. The mean value of this uniform region indicates the true isotope concentration. Recovery coefficients (RC) were calculated on the image slices covering the central $10 \mathrm{~mm}$ length of the rods, which were averaged to obtain a single slice of lower noise. Circular regions of interest (ROIs) were drawn on this image around each rod with diameters twice the rods' physical diameter. The maximum values in each of these ROIs were recorded and used to create line profiles along the axial direction the rods. The isotope concentration was measured from the mean pixel value along each of the profiles in the axial direction. The standard deviation was calculated as:

$$
\left.\% \mathrm{STDRC}=100 * \sqrt{\left(\frac{S T D_{\text {lineprofile }}}{\text { Mean } \text { lineprofile }_{\text {in }}}\right)^{2}+\left(\frac{\text { STD } \text { background }}{\text { Mean }}\right)_{\text {background }}}\right)^{2}
$$

Attenuation correction was evaluated by defining VOIs of $4 \mathrm{~mm}$ in diameter and $7.5 \mathrm{~mm}$ in length in the water- and air-filled cylindrical inserts of the image quality phantom. The ratio of the mean in each cold region to the mean of the hot uniform area was reported as the spill-over ratio (SOR). The standard deviation was calculated in the same way as that of the RCs.

\section{RESULTS}

Spatial Resolution At the center of the FOV the radial, tangential and axial resolutions in terms of FWHM (mm) were $2.12 \mathrm{~mm}, 2.66 \mathrm{~mm}$ and $2.23 \mathrm{~mm}$ respectively. Figure 1 shows the results obtained according to the NEMA NU 4-2008 standards for the spatial resolution of the microPET R4 system in terms of FWHM (Fig. 1A) and FWTM (Fig. 1B). Fig. 2 represents the volumetric resolution of the system.

Scatter fraction, count losses and random coincidence measurements 
Fig. 3 shows the counting rate performance of the microPET R4 scanner. The scatter fraction was $8.5 \%$ for the mouse-sized phantom, while the peak count rate was $311 \mathrm{kcps}$ at $153.5 \mathrm{MBq}$ (Fig. 3A). In the case of the rat-sized phantom the scatter fraction was $22 \%$ and the peak count rate was $117 \mathrm{kcps}$ at $123.24 \mathrm{MBq}$ (Fig. 3B).

Sensitivity The absolute system sensitivity at the center of the FOV was measured to be $1.9 \%(16.66 \mathrm{cps} / \mathrm{kBq})$ for sinograms encompassing the central $7 \mathrm{~cm}$ (mouse-sized phantom) and $15 \mathrm{~cm}$ (rat-sized phantom). The axial FOV of the microPET R4 is $7.8 \mathrm{~cm}$ and therefore sinograms of the rat-sized phantom encompassed the same axial length as the sinogram of the mouse-sized phantom. Figure 4 displays the axial absolute sensitivity profile along the z-axis of the microPET R4 scanner.

Image Quality The reconstruction of the NEMA image quality phantom (Fig. 5) took place using the three reconstruction algorithms provided with the microPET R4 scanner. The pixel size was $0.84 \mathrm{~mm}$ (radial and axial) and in 2D FBP no axial filter was used. Table I summarizes the uniformity results without attenuation correction and with transmission and CT attenuation correction.

The recovery coefficients in function of the rod diameters are represented in Fig. 6.

The spill-over ratios measured in the air- and water-filled cylindrical inserts of the NEMA image quality phantom are indicated in Table 2 for data without correction of attenuation and with transmission-based and CT attenuation correction. The ratio of the reconstructed count density to the true count density of the ROI and the Standard Deviation (\%STD) was estimated from the water and air-filled compartments of the image quality phantom. The results shown below represent the spill-over ratio and the $\%$ STD without correction of attenuation, with $68 \mathrm{Ge}$ transmission based attenuation correction (TX-AC) and with $\mathrm{CT}$ attenuation correction (CT$\mathrm{AC})$.

\section{DISCUSSION}

The performance evaluation of the microPET R4 scanner in function of spatial resolution, sensitivity, scatter fraction, count rate performance and image quality according to the NEMA NU 4-2008 standards for small animal PET systems has been studied.

The system's spatial resolution in the tangential direction was constant but it slightly deteriorated after $10 \mathrm{~mm}$ radial distance when moving away from the CFOV. This finding can be attributed to the parallax error, which causes degradation of the radial resolution at radial offsets. This wellknown geometric error, also referred to as depth of interaction (DOI), derives from the determination of the exact position at which the photon 
hits the crystal, so crystal penetration is more likely to occur for photons with an increasing incident angle [26]. The axial resolution also deteriorated at the end of the FOV ranging from $2.55 \mathrm{~mm} \mathrm{FWHM}$ at $5 \mathrm{~mm}$ radial distance to $3.23 \mathrm{~mm}$ FWHM at $25 \mathrm{~mm}$ radial distance. Due to the fact that the maximum ring difference of the microPET R4 scanner was used to histogram the 3D sinograms to $2 \mathrm{D}$ sinograms and then FORE + 2D FBP reconstruction algorithm was used, may explain these results [27]. The system's volumetric resolution was found to be $11.55 \mathrm{~mm}^{3}$ at the CFOV and $15.08 \mathrm{~mm}^{3}$ at $5 \mathrm{~mm}$ radial distance. Our results of spatial resolution do not significantly depart from those obtained by Knoess et al [21].

Generally, the spatial resolution of a PET system is measured with a point source placed at various distances across the whole FOV. The reproduction of the spatial details in the image is expressed through the point spread function (PSF) and after reconstruction profiles are fitted through the reconstructed image normally with a Gaussian spread function fitted to the PSF. Then the width at half of the maximum value of the PSF, and at tenth of the maximum value of the PSF, is measured. It has to be highlighted that the spatial resolution depends on the shape and the material of the source, the isotope used and the reconstruction method followed. There are various reconstruction methods used in PET ranging from simple 2D and 3D FBP to iterative methods such as ML-EM. Rebinning methods are also used referring either to the axial rebinning of the data (SSRB) or multislice rebinning or Fourier rebinning (FORE) [28].

According to the NEMA NU 4-2008 standards, the proposed methodology for the evaluation of the spatial resolution should be done by 2D or 3D FBP reconstruction with no smoothing applied. In this aspect, it should be considered that one of the major limitations of FBP is the statistical noise, so a smoothing filter should be applied prior to reconstruction in order to control it. Of course, on the other hand, in case of applying a smoothing filter, resolution would degrade because of the reduction of variance in the image [29]. This did not take place during the processing of the spatial resolution and therefore the effect of noise in FWHM and FWTM may explain the relatively high variability in our measurements. Furthermore, NEMA NU 4-2008 standards indicate that the FWHM should span at least five pixels; some scanners are unable to fulfill this requirement and therefore cannot follow the same pattern for the performance evaluation [30].

The sensitivity of the system was measured to be $1.9 \%$ at the center of the scanner's FOV using an energy window of 350-650 keV. This was calculated using the rate of emitted prompt gamma rays, meaning the true, scatter and random coincidences, coming from the ${ }^{22} \mathrm{Na}$ point source to the detectors. The axial sensitivity profile drops linearly from the center to the 
edges of the FOV. The presence of the intrinsic radioactivity of LSO $\left({ }^{176} \mathrm{Lu}\right)$ affects the system's ability to detect low activities in the FOV. In the microPET R4 scanner the activity of ${ }^{176} \mathrm{Lu}$ in the $271 \mathrm{~cm}^{3}$ of LSO is approximately $75 \mathrm{kBq}$ [31] but the fact that an energy window of 350-650 $\mathrm{keV}$ was used, significantly reduced the amount of the intrinsic count rate and its contribution to the statistical noise.

Knoess et al., [21] followed a totally different methodology to evaluate the sensitivity of the microPET R4 scanner. They used a ${ }^{68} \mathrm{Ge}$ line source and aluminium sleeves with different diameters and wall thicknesses to create a shielding around the source for various energy windows. Sensitivity was calculated by plotting the true count rate against the total shielding thickness and by extrapolating to the equivalent unshielded line source, with no attenuation to the true count rate. System sensitivity for an energy window of $350-650 \mathrm{keV}$ was found to be $12.24 \mathrm{cps} / \mathrm{kBq}(1.37 \%)$. They also performed an additional measurement using a ${ }^{22} \mathrm{Na}$ point source centered in the transaxial direction of the FOV. The radial direction was scanned from $0 \mathrm{~mm}$ to $50 \mathrm{~mm}$ offset in steps of $5 \mathrm{~mm}$ yielding a sensitivity of $24.48 \mathrm{cps} / \mathrm{kBq}(2.7 \%)$ for an energy window of $350-650 \mathrm{keV} \mathrm{[21].}$

The counting rate was plotted as a function of the average activity concentration for the mouse- and rat-sized phantoms. Its maximum corresponds to the relationship between real and noise events. After reaching its maximum it slowly decreases as the number of random coincidences increases due to dead time effects. The peak NEC rate was found to be $311 \mathrm{kcps}$ at $153.5 \mathrm{MBq}$ for the mouse-sized phantom and 117 kcps at $123.24 \mathrm{MBq}$ for the rat-sized phantom. The NECR for the mousesized phantom was found to be higher than the NECR for the rat-sized phantom because there is less photon attenuation forcing more photons getting out of the imaging subject. According to Knoess et al. [21] the maximal NEC ratio for the mouse phantom was $168 \mathrm{kcps}$ at $91 \mathrm{MBq}$ and for the rat phantom it was $89 \mathrm{kcps}$ at $81 \mathrm{MBq}$ for an energy window of 350$750 \mathrm{keV}$.

During the evaluation of the scatter fraction and count rate performance according to the NEMA NU 2-2001 [21], bigger phantoms were used as well as different energy windows [32]. For example, according to Knoess et al. [21] scatter fraction values were 18\% (mouse-sized phantom) and $28 \%$ (rat-sized phantom) for an energy window of 350-750 keV, whereas in this work they were reduced to $8.5 \%$ and $22 \%$ respectively for an energy window of 350-650 keV. Since the ratio of rays falling into the range of $650-750 \mathrm{keV}$ is expected to be very low, these differences in the results can be safely attributed to the different phantom sizes used in the experiments.

The image quality results provide estimation for a standardized imaging situation in small animal PET systems. All images were reconstructed using 
the three reconstruction algorithms provided by the microPET R4 scanner. Correction of attenuation was performed in order to assess the impact of corrections in the measurements, especially when cold regions are used where scatter radiation is increased. It is known though that the problem of photon attenuation in small animal imaging is not so much of importance since the size of the animals is much lower than in the human case.

In the case of uniformity, the \%STD indicated better results when using FBP reconstruction algorithm than the two iterative methods. This may be surprising but it concurs with Bahri M. et al [33] who found that the mean activity was within $99 \%$ of the expected value for FORE reconstruction methods.

However, an overestimation of the recovery coefficient in large rods of $5 \mathrm{~mm}$ in diameter was obtained with 2D and 3D OSEM MAP reconstruction methods. This behavior might bias image quantification. It should be noted that since the spatial resolution of the system was over $2 \mathrm{~mm}$, the maximum cylinder diameter made it impossible to fully recover the total activity inside. The expected RC for a cylinder with a diameter 2.37 times the FWHM of the system is of about 0.95 [34]. The spill over ratio decreased with the use of 2D OSEM reconstruction algorithm. This can be attributed to an improvement in spatial resolution with iterative methods as compared to FBP. SOR values for the air compartment of the phantom were close to zero with or without attenuation correction.

When using the ${ }^{68} \mathrm{Ge}$ based transmission attenuation correction method, there was no significant change in the SOR values apart from a slight decrease in the phantom's water compartment. The CT based attenuation correction method used in this study provided lower statistical noise and higher resolution anatomical images in comparison to the TX-AC method. A significant decrease in the SOR values of the water compartment was observed while in the air compartment values remained close to zero. No scatter correction method was applied in this study.

The evaluation of the image quality after reconstruction of the images was first introduced in NEMA NU 4-2008 standards from small animal PET systems, so no previous results exist so as to be compared with the ones obtained here.

It is worth mentioning that NEMA standards try to provide a standardized methodology for evaluating the performance of small animal PET systems and that these measurements are not absolute. They can be used for comparing different systems since the methodology provided is fairly easy to perform although there are some ambiguities in some sections of the NEMA NU 4-2008 standards. For example, it should be made clearer when calculating the sensitivity of a system as to which counts can be used when a system provides prompts and histogrammed coincidences as in the case 
of the microPET R4 scanner. Also, the quality of the reconstructed image depends on the size of the object and the contrasts used. The size of the NEMA NU 4-2008 image quality phantom is more representative of a mouse's body and a rat's head; therefore the results obtained under these specifications must take into account the above statement.

Furthermore, NEMA NU 4- 2008 standards are not so appropriate for evaluating the performance of scanners that do not employ conventional cylindrical geometry, so modifications are required in order to provide meaningful results as in the case of the PETbox scanner [35].

\section{CONCLUSIONS}

This study evaluated the performance of the microPET R4 system according to the new NEMA NU 4-2008 standards for small animal PET systems and compared it with its previous evaluation according to [21]. Our findings highlight the importance of using specific performance evaluation tests for small animal imaging as some of the performance indicators calculated herein in accordance with the new standards diverge notably from published results using older ones.

To summarize our findings regarding reconstruction algorithms, FBP provided the best uniformity, at the expense of a mild underestimation of absolute activity concentration and the worst spatial resolution. 2D-OSEM yielded the best quantification accuracy and slightly worse uniformity than FBP. Finally, 3D-OSEM resulted in an important positive bias in quantitative measurements and the worst uniformity of the three evaluated methods, although it provided the best results in terms of spatial resolution. Here, when values are compared to previously results, variations can be noticed which could be attributed to the fact that different phantoms and energy windows were used.

\section{ACKNOWLEDGMENT}

The authors kindly thank Dr. Juan José Vaquero for providing the phantoms for the system's count rate and scatter fraction. We also thank Ma Dolors de la Fuente for her valuable help and technical support.

\section{REFERENCES}

[1] S. R. Cherry, "Fundamentals of Positron Emission Tomography and applications in preclinical drug development", J. Clin Pharmacol., vol. 41, pp. 482-91, 2001.

[2] M. E. Phelps, "Positron emission tomography provides molecular imaging of biological processes", Proc Natl Acad Sci., vol. 97, pp. 9226-9233, 2000. 
[3] C. S. Levin, "Primer on molecular imaging technology", Eur J Nuc Med Mol Imaging, vol. 32(14), pp. S325-S345, 2005.

[4] C. S. Levin, H. Zaidi, "Current trends in preclinical PET system design", PET Clin. vol. 42, pp. 219-232, 2007.

[5] H. Zhang, S. Alyafei, T. Inoue, K. Tomiyoshi, K. Endo, "Performance stability of SHR 2000 high resolution PET for small animal research", Annals of Nucl Med, vol. 13(1), pp. 65-70, 1999.

[6] D. P. Cutler, S. R. Cherry, E. J. Hoffman, W. M. Digby, M. E. Phelps, "Design features and performance of a PET system for animal research", J Nuc Med, vol. 33, pp. 595-604, 1992.

[7] S. R. Cherry, Y. Shao, S. Siegel, R.W. Silverman, K. Meadors, J. Young, et al., "MicroPET: a high resolution PET scanner for imaging small animals", IEEE Trans Nuc Sci., vol. 44, pp. 1161-1166, 1997.

[8] A. Del Guerra, G. Di Domenico, M. Scandola and G. Zavattini, "YAPPET: first results of s small animal positron emission tomography based on YAP:Ce finger crystals", IEEE Trans Nucl Sci. vol. 45, pp. 31053108, 1998.

[9] A.P. Jeavons, R.A. Chandler and CAR. Dettmar, "A 3D HIDAC-PET camera with sub millimetre resolution for imaging small animals", IEEE Trans Nucl Sci, vol. 46, pp. 468-473, 1999.

[10] C. Tai, A. Chatziioannou, S. Siegel, J. Young, D. Newport, R.N. Goble, R.E. Nutt and S.R. Cherry, "Performance evaluation of the microPET P4: a PET system dedicated to animal imaging", Phys Med Biol., vol. 46, pp. 1845-1862, 2001.

[11] P. Vasca, C.L. Woody, D.J. Schlyer, S. Shokouhi, S.P. Stoll, J. F. Pratte, et al., "RatCAP: Miniaturized head-mounted PET for conscious rodent brain imaging", IEEE Trans Nucl Sci., vol. 51(5), pp. 27182722, 2004.

[12] National Electrical Manufactures Association. Performance Measurements of Positron Emission Tomogaphs., Rosslyn VA, 1994. Standards Publication NU 2- 1994.

[13] National Electrical Manufactures Association. Performance Measurements of Positron Emission Tomogaphs., Rosslyn VA; 2001. Standards Publication NU 2-2001.

[14] J. Missimer, Z. Madi, M. Honer, C. Keller, A. Schubiger and S.M. Ametamey, "Performance evaluation of the 16-module quadHIDAC small animal PET camera", Phys Med Biol,.vol. 49, pp. 20692081, 2004.

[15] National Electrical Manufactures Association. Performance Measurements of Positron Emission Tomogaphs. Rosslyn VA; 2008. Standards Publication NU 4-2008.

[16] Q. Bao, D. Newport, M. Chen, D.B. Stout and A.F. Chatziioannou, "Performance evaluation of the Inveon dedicated PET preclinical 
tomograph based on the NEMA NU 4- standards", J Nuc Med., vol. 50, pp. 401-408, 2009.

[17] R. Prasad, O. Ratib and H. Zaidi, " Performance evaluation of the Flex Triumph X-PET scanner using the National Electrical Manufacturers Association NU-4 standards", J Nuc Med., vol. 51, pp. 1608-1615, 2010.

[18] S.A. Kis, I. Latjos, M. Emri, L. Tron, G. Opposits, T. Bukki, et al., "Performance test of the MiniPET-II small animal scanner according to the NEMA NU-4 standards", IEEE NSS Conference Record, M09-152, 2009.

[19] M.A. Bahri, A. Plenevaux, G. Warnock, A. Luxen and A. Seret, "NEMA NU4-2008 image quality performance report for the microPET Focus 120 and for various transmission and reconstruction methods", J Nuc Med., vol. 50, pp. 1730-1738, 2009.

[20] E. Lage, J.J. Vaquero, A. Sisniega, S. España, G. Tapias, M. Abella, et al., "Design and performance evaluation of a coplanar multimodality scanner for rodent imaging", Phys Med Biol., vol. 54, pp. 5427-5441, 2009.

[21] C. Knoess, S. Siegel, A. Smith, D. Newport, N. Richerzhagen, A. Winkeler, et al., "Performance evaluation of the microPET R4 scanner for rodents", Eur J Nuc Med Mol Imaging., vol. 30, pp. 737-747, 2003.

[22] C.L. Melcher and J.S. Schweitzer, "Cerium-doped Lutetium Oxyorthosilicate: A Fast, Efficient New Scintillator”, IEEE Trans Nuc Sci., vol. 39(4), pp. 502-505, 1992.

[23] F. Daghighian, P. Shenderov, K.S. Pentlow, M.C. Graham, C.L. Melcher and J.S Schweitzer, "Evaluation of Cerium Doped Lutetium Oxyorthosilicate (LSO) Scintillation crystal for PET", IEEE Trans Nuc Sci., 1045-1047, 1993.

[24] S. Siegel, M. Eriksson, L. Eriksson, M. Casey and R. Nutt, "An alternative to polishing the surfaces of scintillation detectors", IEEE MIC Conference Record. , pp. 1212-1214, 2000.

[25] M. Titeler, R.A. Lyon, M.J. Kuhar, J.F. Frost, R.F. Dannals, S. Leonhardt, et al., "Mu opiate receptors are selectively labelled by [3H] carfentanil in human and rat brain", Eur J Pharmacol., vol. 22, pp. 221$8,1989$.

[26] J. Seidel, J.J. Vaquero and M.V. Green, "Resolution uniformity and sensitivity of the NIH ATLAS small animal PET scanner: Comparison to simulated LSO scanners without Depth-Of-Interaction capability", IEEE Trans Nuc Sci., vol. 50(5), pp. 1347-1350, 2003.

[27] E.P. Visser, A. Disselhorst, M. Brom, P. Laverman, M. Gotthardt, W.J. Oven and O.C. Boerman, "Spatial Resolution and Sensitivity of the Inveon Small Animal PET scanner", J Nuc Med., vol. 50, pp. 139147, 2009. 
[28] S. Weber and A. Bauer, "Small animal PET: aspects of performance assessment", Eur J Nuc Med and Mol Imaging., vol. 31(11), pp. 1545-1555, 2004.

[29] A. Alessio and P. Kinahan, "PET Image reconstruction", in: Henkin RE, Bova D, Dillehay GL, Halama JR, Karesh SM, Wagner RH, Zimmer AM, eds. Nuclear Medicine. 2nd ed., vol. 1, Philadelphia, PA: Mosby-Elsevier; 2006.

[30] J.J. Vaquero, E. Lage, L. Ricon, E. Abella, E. Vicente and M. Desco, "rPET detectors design and data processing", IEEE Nuc Sci Symp Conf Rec., pp. 2885-2889, 2005.

[31] A.L. Goertzen, J.Y. Suk and C.J. Thompson, "Imaging of WeakSource Distributions in LSO-Based Small Animal PET scanners", $J$ Nucl Med. vol. 48, pp. 1692-1698, 2007.

[32] F.D. Popota, P. Aguiar, Y. Fernandez, C. Lois, D. Pareto, D. Ros, et al., "Comparison of NEMA NU 4-2008 vs NEMA NU 2-2001 for the performance evaluation of the microPET R4 system", IEEE Nucl Sci Symp Conference Record., M05-151, pp. 2706-2709, 2009.

[33] M.A. Bahri, A. Plenevaux, G. Warnock, A. Luxen and A. Seret, "NEMA NU 4-2008 Image quality performance report for the microPET Focus 120 and for various transmission and reconstruction methods", J Nucl Med. vol. 50, pp. 1730-1738, 2009.

[34] S. R. Cherry and M. Dalhbom, "PET: Physics, Instrumentation and Scanners", in: Phelps M, eds. PET Molecular Imaging and its Biological Applications, New York, NY: Springer Verlag Inc.; 2004.

[35] H. Zhang, Q. Bao, T.N. Vu, R.W. Silverman, R. Taschereau, B.N. Berry-Pusey, A. Douraghy, F.R. Rannou, D.B. Stout and A.F. Chatziioannou, "Performance evaluation of PETBox: A low cost bench top preclinical PET scanner" [research article], Mol Imaging Biol. vol. 13(5), pp. 949-961, 2011. 


\section{FIGURES:}
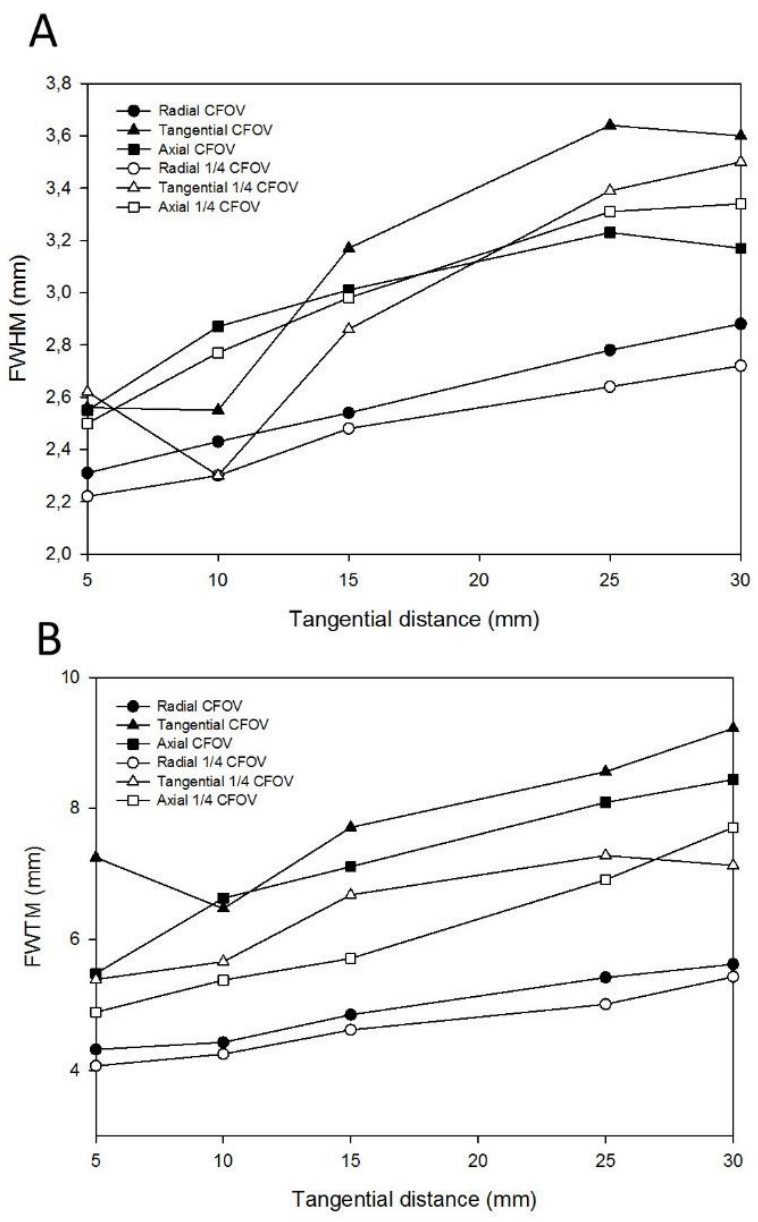

Fig. 1 Spatial resolution as a function of radial offset in terms of FWHM

(A) and FWTM (B) 


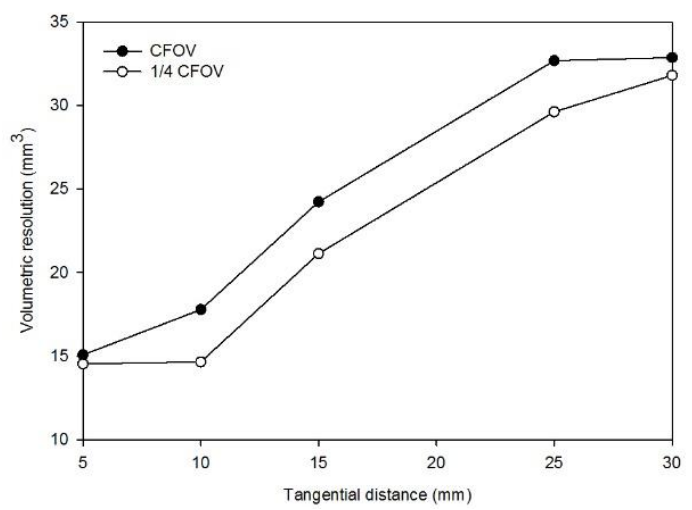

Fig. 2 Volumetric resolution at axial center and at $1 / 4$ of the axial center
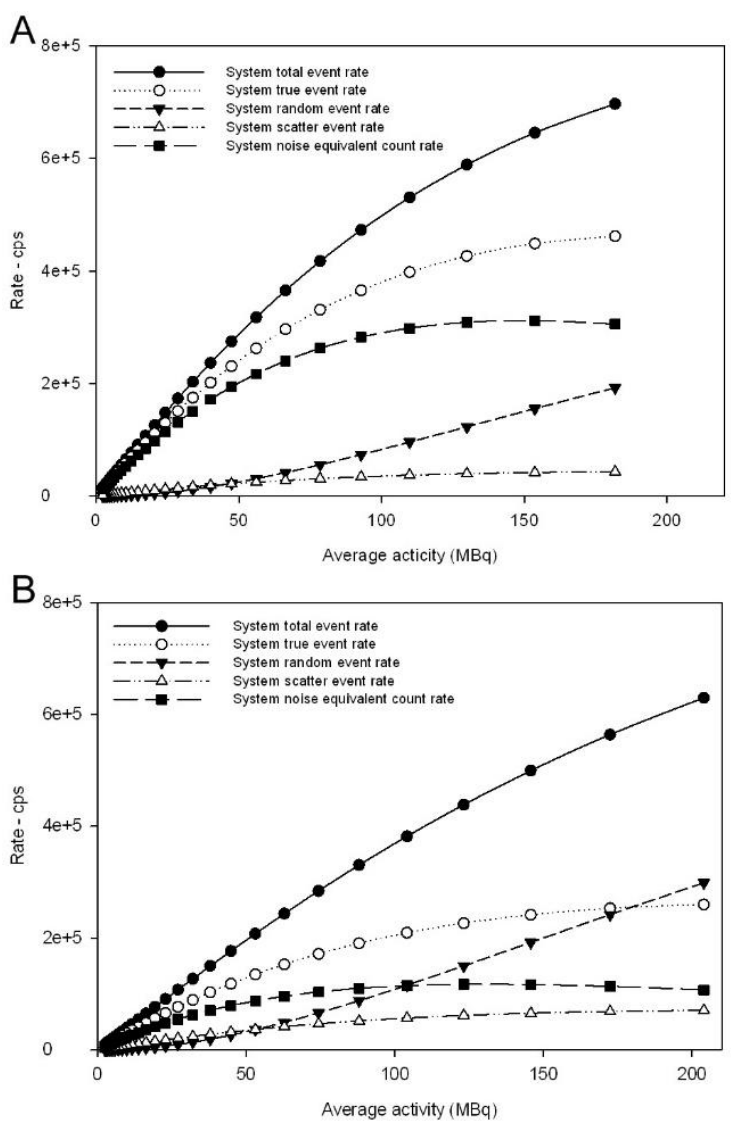

Fig. 3 Count rate performance for mouse-sized (A) and rat-sized (B) phantoms 


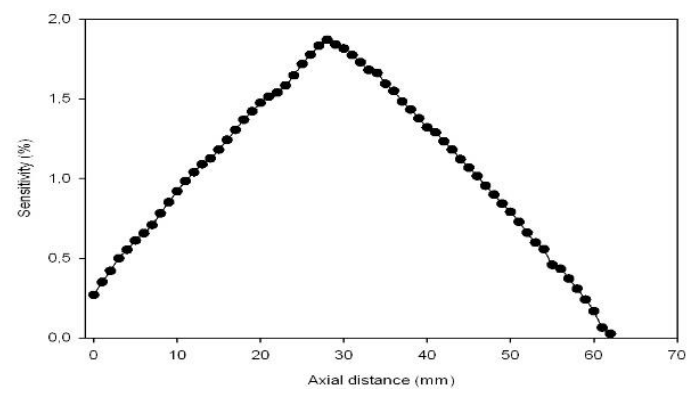

Fig. 4 Axial sensitivity profile

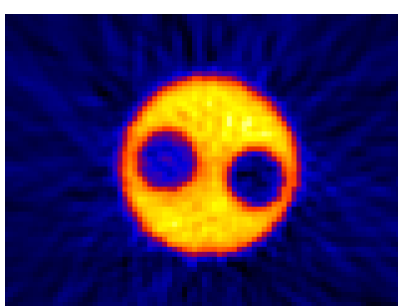

(a)

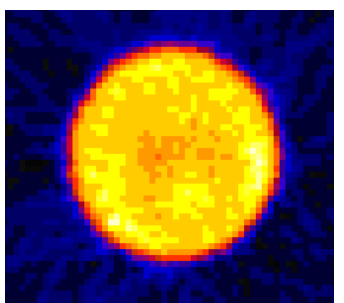

(b)

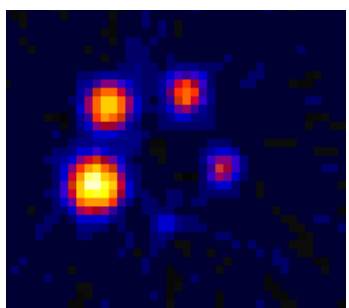

(c)

Fig. 5 microPET R4 transverse views of image reconstruction of the quality phantom; a) transverse view of the non-radioactive water and air chambers, b) transverse view of the uniform part and c) transverse view of the five cylindrical rods

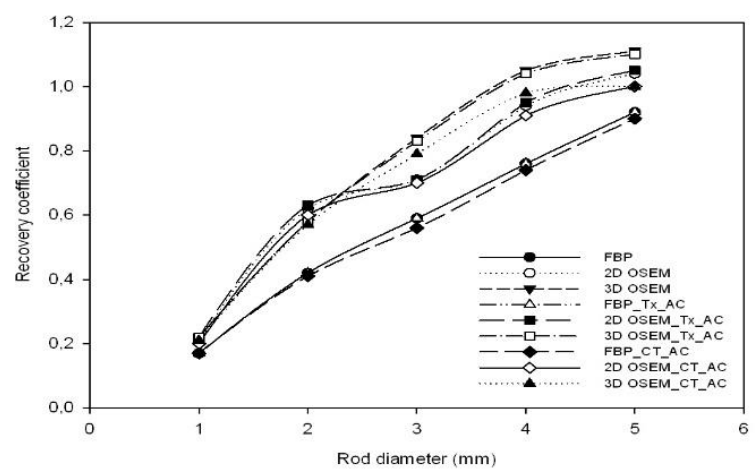

Fig. 6 Recovery coefficients using the three reconstruction algorithms of the microPET R4 with and without corrections 


\begin{tabular}{|l|r|r|r|r|}
\hline $\begin{array}{l}\text { Reconstruction } \\
\text { algorithms }\end{array}$ & $\begin{array}{l}\text { Mean } \\
\text { (nCi/cc) }\end{array}$ & $\begin{array}{l}\text { Max } \\
\text { (nCi/cc) }\end{array}$ & \multicolumn{1}{l|}{$\begin{array}{l}\text { Min } \\
\text { (nCi/cc) }\end{array}$} & \%STD \\
\hline FBP & 4533.75 & 5693.45 & 2970.93 & 7.76 \\
\hline 2D OSEM & 4534.46 & 5779.23 & 2953.87 & 8.67 \\
\hline 3D OSEM & 4788.14 & 6351.49 & 3343.52 & 8.92 \\
\hline FBP_TX_AC & 4681.77 & 5741.85 & 3076.43 & 7.63 \\
\hline 2D OSEM_TX_AC & 4680.87 & 5997.99 & 3060.82 & 8.56 \\
\hline 3D OSEM_TX_AC & 4949.35 & 6618.83 & 3486.1 & 8.81 \\
\hline FBP_CT_AC & 6959.03 & 8753.27 & 4216.77 & 7.28 \\
\hline 2D OSEM_CT_AC & 6957.2 & 9106.16 & 4152.94 & 8.28 \\
\hline 3D OSEM_CT_AC & 7369.77 & 9960 & 4849.67 & 9.52 \\
\hline
\end{tabular}

TABLE I Uniformity values for the microPET R4 with and without attenuation corrections

\begin{tabular}{|c|c|c|c|c|c|}
\hline & & \multicolumn{2}{|c|}{ Water } & \multicolumn{2}{|c|}{ Air } \\
\hline & & SOR & \%STD & SOR & $\%$ STD \\
\hline \multirow{3}{*}{$\begin{array}{c}\text { No } \\
\text { attenuation } \\
\text { correction }\end{array}$} & FBP & 0.23 & 38.86 & 0.05 & 46.27 \\
\hline & $\begin{array}{c}\text { OSEM } \\
\text { 2D }\end{array}$ & 0.21 & 41.82 & 0.09 & 43.69 \\
\hline & $\begin{array}{c}\text { OSEM } \\
\text { 3D }\end{array}$ & 0.23 & 58.29 & 0.01 & 123.21 \\
\hline \multirow{3}{*}{$\begin{array}{c}\mathbf{T x} \\
\text { attenuation } \\
\text { correction }\end{array}$} & FBP & 0.21 & 38.01 & 0.05 & 43.93 \\
\hline & $\begin{array}{c}\text { OSEM } \\
\text { 2D } \\
\end{array}$ & 0.20 & 42.64 & 0.08 & 42.96 \\
\hline & $\begin{array}{c}\text { OSEM } \\
\text { 3D }\end{array}$ & 0.22 & 57.76 & 0.01 & 73.79 \\
\hline \multirow{3}{*}{$\begin{array}{c}\mathrm{CT} \\
\text { attenuation } \\
\text { correction }\end{array}$} & FBP & 0.09 & 59.4 & 0.06 & 62.29 \\
\hline & $\begin{array}{c}\text { OSEM } \\
\text { 2D }\end{array}$ & 0.15 & 44.18 & 0.10 & 44.12 \\
\hline & $\begin{array}{c}\text { OSEM } \\
\text { 3D } \\
\end{array}$ & 0.02 & 99.53 & 0.01 & 68.44 \\
\hline
\end{tabular}

TABLE II SOR and \%SDT for all reconstruction algorithms from the microPET R4 scanner without corrections and with transmission and CT attenuation correction 